\title{
Efficacy and Safety of Xuebijing Injection for Severe Pneumonia: A Protocol for A Systematic Review and Meta-Analysis
}

\section{Mina Zhang}

Beijing Hospital of Traditional Chinese Medicine;Beijing Institute of Traditional Chinese Medicine;Beijing University of Chinese Medicine https://orcid.org/0000-0002-2755-624X

\section{Luda Feng}

Beijing University of Chinese Medicine;Beijing University of Chinese Medicine Affiliated Dongzhimen Hospital;Institute for Brain Disorders,Beijing University of Chinese Medicine

\section{Shuwen Zhang}

Beijing Hospital of Traditional Chinese Medicine;Beijing Institute of Traditional Chinese Medicine;Beijing university of Chinese Medicine

\section{Ping Jiang}

Beijing University of Chinese Medicine;Beijing University of Chinese Medicine Affiliated Dongzhimen Hospital;Institute for Brain Disorders,Beijing University of Chinese Medicine

\section{Ning Liang}

Institute of Basic Research in Clinical Medicine,Chaina Academy of Chinese Medicine Sciences

\section{Po Huang}

Beijing Hospital of Traditional Chinese Medicine

\section{Xiaolong Xu}

Beijing Hospital of Traditional Chinese Medicine;Beijing Institute of Traditional Chinese Medicine

Qingquan Liu ( $\square$ liuqingquan_2003@126.com)

Beijing Hospital of Traditional Chinese Medicine;Beijing Institute of Traditional Chinese Medicine;Beijing University of Chinese Medicine https://orcid.org/0000-0003-0828-0361

\section{Protocol}

Keywords: efficacy, randomized controlled trial, safety, severe pneumonia, xuebijing injection

Posted Date: November 18th, 2020

DOl: https://doi.org/10.21203/rs.3.rs-107413/v1

License: (c) (i) This work is licensed under a Creative Commons Attribution 4.0 International License. Read Full License 


\section{Abstract}

Background: Severe pneumonia (SP) is a life-threatening condition without specific treatment options. Although there are many available treatment options for SP, most of the therapies have been associated with mild or severe side effects. The purpose of this meta-analysis is to examine the efficacy and safety of Xuebijing injection (XBJ), a Chinese patent approved by the China Food and Drug Administration, for treating patients with SP.

Methods: An electronic and manual search of published articles, ongoing trials, dissertations, and grey literature will be performed. Electronic databases including Medline, Embase, the Cochrane Central Register of Controlled Trials (CENTRAL), China National Knowledge Infrastructure (CNKI), China Science and Technology Journal Database (VIP), Wanfang Data, SinoMed will be searched without language restrictions from their inception to 01/10/2020. All randomized controlled trials where (a) XBJ is compared with placebo or no treatment, (b) XBJ plus routine treatment is compared with routine treatment, and (c) XBJ plus co-interventions are compared with co-interventions will be included. Severe pneumonia-related mortality will be set as the primary outcome. Study inclusion, data extraction, and quality assessment will be independently performed by two reviewers. Review Manager 5.3 is to be used to perform the assessment of the risk of bias, data synthesis, and subgroup analysis. Grading of Recommendations Assessment, Development, and Evaluation approach will be used to evaluate the quality of the overall evidence. This protocol was performed in accordance with the Preferred Reporting Items for Systematic Review and Meta-Analysis Protocols.

Discussion: This proposed systematic review and meta-analysis will identify and synthesise evidence to clarify efficacy and safety of XBJ for SP. The results will help provide the best available evidence to decision-makers.

\section{PROSPERO registration number. CRD42020181234}

\section{Background}

Severe pneumonia (SP) is a serious complication of respiratory system diseases with high mortality (30\%-50\%) and causes extremely large burden of disease around the world ${ }^{1}$. Commonly, SP develops following community-acquired pneumonia (CAP), hospital-acquired pneumonia (HAP), and ventilatorassociated pneumonia (VAP) ${ }^{1}$.

The complex pathological mechanisms of SP lead to several severe complications (acute respiratory distress syndrome, system inflammatory reaction syndrome, sepsis, coagulation dysfunction, and multiorgan failure). Once in the body, pathogenic microorganisms can directly damage the structure and function of the lung. These changes accelerate the production of large numbers of inflammatory mediators. When pro-inflammatory and anti-inflammatory reactions are out of balance, the "waterfall" cascade of inflammation will occur in serum ${ }^{2}$. Additionally, the inflammatory mediators and proinflammatory cytokines activates the coagulation pathway, and then, the severe coagulopathy furtherly 
aggravates the inflammatory injury in return. This vicious circle acts a crucial role in the pathogenesis mechanism of SP, worsening the conditions and contributing to death ${ }^{3}$. Moreover, excessive stress response of the body leads to the disruption of the balance of redox reaction, and then produce oxygen free radicals, which induce oxidative damage to cells ${ }^{4}$. The inflammatory overreaction induced the immune dysfunction also affects the body's self-regulation ${ }^{5}$.

Over the last decade, great progress in antibiotic and life-supportive treatments has been made. Under conventional anti-infection treatment and symptomatic support treatment, SP remains a leading cause of admission and death in ICU due to its complexity ${ }^{6}$. Some researchers resort to traditional Chinese medicine due to conventional therapies did not significantly improve the survival rate after SP. Xuebijing injection (XBJ), a Chinese patent, was approved by the China Food and Drug Administration for the treatment of sepsis in critically ill patients, ${ }^{7}$ and it has been identified as a promising treatment approach for SP. XBJ was modified from Xuefu Zhuyu Decoction, a classic prescription created in the Qing dynasty. $\mathrm{XBJ}$ can promote blood circulation and remove blood stasis, as well as mediate fever attenuation and detoxification. This compound injection is composed of five Chinese herbs. As the sovereign drug, honghua (safflower) can activate blood and remove stasis; chishao (red peony root) and chuanxiong (ligusticum wallichii) are drugs that can magnify the effects of the sovereign drug, cool blood, remove stasis, move qi and that have a detoxifying effect; danshen (salvia miltiorrhiza) and danggui (angelica sinensis) act as supporters that enrich blood and remove stasis.

In long-term clinical practice, $\mathrm{XBJ}$ has been successfully used to improve $\mathrm{SP}^{8-10}$. Moreover, preclinical studies have shown that XBJ might exert therapeutic effects by multiple mechanisms. XBJ has a positive role in preventing cytokine storm, controlling inflammation, regulating immunity, and improving multiple metabolic pathways ${ }^{11-13}$. In clinical studies, XBJ can relieve symptoms, increase the clinical cure rate, and decrease 28 -day mortality ${ }^{9}$. It also shows anti-bacterial and anti-endotoxin effect ${ }^{14}$. Furthermore, it can also alleviate excessive inflammatory response, ameliorate coagulopathy ${ }^{15}$, improve microcirculation ${ }^{16}$, modulate immune function ${ }^{17}$, and protect organ function ${ }^{18}$. These findings indicate that XBJ has many advantages over other treatments in the treatment of SP by multi-target and multipathway.

Although previous randomized controlled trials (RCTs) reported that XBJ could be used to treat SP effectively, samples used in these RCTs were too limited to determine the robustness of the findings. Over the past 5 years, both the number and quality of clinical trials using XBJ to treat SP have improved considerably. Emerging evidence from some RCTs suggested that XBJ may be effective in treating SP by not increasing mortality and incidence of side effects, as well as by improving clinical symptoms and reinforcing the effectiveness of antibiotics ${ }^{19,20}$. And a multi-center, large-scale clinical trial evaluated the efficacy of XBJ versus placebo in treating SCAP, demonstrating XBJ to be effective and safe for SP9 ${ }^{9}$ Yet, the safety of XBJ remains controversial. Results of different real-world studies indicated the common adverse drug reactions include skin pruritus, erythra, and chest tightness, which were associated with vehicle type, dose, and drug combinations ${ }^{21} 22$. Besides, it is crucial to evaluate the specifics of XBJ for 
SP, which include the optimal dose, the right time to start treatment and the duration of treatment. What is noteworthy is that despite more than 100 clinical trials have been reported in the recent 5 years, XBJ as a therapeutic drug for SP remains in dispute. Furthermore, the mortality rate, severity of pneumonia and the impairment of multiple organs are vital clinical efficacy indicators of SP. Nevertheless, previous systematic review and meta-analysis neither demonstrated convincing data on mortality decrease nor reported analysis on improvements in pneumonia severity or multi-organ impairment ${ }^{23-25}$. Considering that some RCTs, assessed mortality and organ function ${ }^{9,19,26}$, it is necessary to update the systematic review and meta-analysis to obtain up-to-date evidence. Under these urgent circumstances, we plan to specifically investigate the efficacy and safety of XBJ in the treatment of SP. Furthermore, this study will try to obtain conclusive evidence in support of XBJ as a proper choice for patients with SP.

\section{Objective}

The aim of this systematic review and meta-analysis is to systematically analyze all the RCTs so as to evaluate the efficacy and safety of XBJ for SP, providing the best available evidence.

\section{Methods/design}

\section{Protocol and registration}

This protocol of systematic review and meta-analysis will be conducted following the Preferred Reporting Items for Systematic Reviews and Meta-Analysis Protocols (PRISMA-P) guidelines ${ }^{27}$. See (Appendix 1) for the completed PRISMA-P checklist.

This protocol is registered in the International Prospective Register of Systematic Reviews (PROSPERO) system and can be accessed at https://www.crd.york.ac.uk/PROSPERO/display_record.php? RecordID $=181234$.

\section{Eligibility criteria}

\section{Type of studies to be included}

All relevant RCTs with or without blinding will be included. The quasi-RCTs, non-RCTs, cross-over study, and uncontrolled clinical trials will be excluded. There will be no language restrictions.

\section{Types of participants}

Adult participants diagnosed as SP according to the Infectious Diseases Society of America/American Thoracic Society guidelines will be inclduded ${ }^{28}$. There will be no limitation on age, gender, education, ethnicity, and economic status. Patients with serious complications will be excluded.

\section{Types of interventions}


On the basis of conventional treatment, the treatment intervention will be XBJ. The control intervention will be based on a placebo or no intervention. Additionally, trials that evaluate XBJ plus another therapy compared with the other therapy alone will also be included.

\section{Types of outcome measures}

\section{(1) Primary outcomes}

Severe pneumonia-related mortality.

\section{(2) Secondary outcomes}

The secondary outcomes will include (a) proportion of total efficiency rate including cure rate, obvious effective rate, and effective rate; (b) improvement in acute physiology and chronic health evaluation II; (c) improvement of pneumonia severity assessed by clinical scales including clinical pulmonary infection score or pneumonia severity index; (d) course of antibiotic use; (e) total duration of ICU stays and hospitalization.

\section{(3) Safety outcomes}

Adverse effects of XBJ include aphylactic reaction, impairment of liver and kidney function, nausea, and skin irritation.

\section{Search strategy}

We will search for the following databases from their inception until 1 October 2020: MEDLINE, EMBASE, CENTRAL, and the following Chinese databases and sources: CNKI, VIP, SinoMed, Wanfang Data. Additionally, we will search for dissertations, clinical trial registers, and grey literature. The following registers for ongoing or unpublished trials will be searched: World Health Organization International Clinical Trials Registry Platform (www.who.int/ictrp), ClinicalTrials.gov (www.clinicaltrials.gov/), and the Chinese Clinical Trial Registry (ChiCTR). The search strategy for Medline will be provided (Appendix 2). We will use the equivalent search words in the Chinese databases as well.

\section{Screening and selection}

According to the inclusion criteria, two review authors (MNZ, LDF) will independently screen the titles and abstracts of all searched studies and exclude reports that are obviously irrelevant. They will retrieve fulltext articles to identify studies for inclusion and record reasons for the exclusion of ineligible studies. Disagreements will be resolved through discussion or by consulting a third author (QQL). The study selection procedure is shown in Figure 1.

\section{Data extraction}


Two review authors (SWZ, PJ) will independently extract data and details from included studies using a preformulated data collection form. Extracted data will be compared by two review authors for completeness and accuracy and double-checked by another review author (PH) if necessary. Disagreements will be solved through discussion with QQL. The data extraction form will include the methods of the study, patients, interventions, duration, and relevant outcomes. If one or more included studies contain missing or unclear information, the authors will be contacted directly.

\section{Assessment of risk of bias in included studies}

The methodological quality of the eligible studies will be independently conducted by two reviewers (MNZ, LDF) for each study using the criteria outlined in the Cochrane Handbook for Systematic Reviews of Interventions. Any disagreements will be solved by involving another review author (QQL). Risk of bias will be assessed according to the following domains: random sequence generation, allocation concealment, blinding of participants and personnel, blinding of outcome assessment, incomplete outcome data, selective outcome reporting, and other bias.

We will grade the risk of bias for each domain as high, low, or unclear, and provide information from the study report, together with a justification for our judgment, in the 'risk of bias' tables.

\section{Data synthesis and management}

\section{Measures of treatment effect}

For continuous data, we will use the mean difference to measure the treatment effect, with a $95 \%$ confidence interval $(\mathrm{Cl})$. Standardized mean difference with $95 \% \mathrm{Cl}$ will be used for when different scales were used to measure a certain outcome variable. For dichotomous data, we will use the risk ratio with $95 \% \mathrm{Cl}$ to measure treatment effects.

\section{Dealing with missing data}

For each included study, missing data and information will be gathered by contacting the study author directly. If the missing data are unavailable, we will perform intention-to-treat and sensitivity analyses to address the potential impact of missing data. If necessary, the potential impact will be described in the 'Discussion' section.

\section{Assessment of heterogeneity and data synthesis}

Whether a fixed-effect model or a random-effect model will be used depends on the results of the $I^{2}$ test for heterogeneity among the trials in each analysis. A meta-analysis will be conducted if the $\mathrm{I}^{2}$ less than $75 \%$ among the included trials. However, a fixed-effect model will be applied to synthesize the data when the $\mathrm{I}^{2}$ value is less than $25 \%$. The sources of heterogeneity will be estimated by sensitive analysis or subgroup analysis when $\mathrm{I}^{2}$ is between $25 \%$ and $75 \%$. If the statistical heterogeneity is successfully explained (i.e., $I^{2}<25 \%$ ), the fixed-effect model will be used to synthesize the data. Otherwise, we will use 
the random-effect model. If there is considerable heterogeneity (i.e., I $^{2} \nabla 75 \%$ ), meta-analysis will not be performed but a systematic narrative synthesis will be conducted. All statistical analyses will be performed using Review Manager V.5.3 software.

\section{Analysis of subgroups or subsets}

Subgroup analyses will be conducted to determine the effects of various dosages, various courses of treatment, and various drug combinations if the data are available.

\section{Assessment of reporting biases}

If ten or more trials are included, we will use funnel plots to assess the presence of publication bias.

\section{Confidence in cumulative evidence}

On the basis of the Grading of Recommendations Assessment, Development, and Evaluation system ${ }^{29}$, the strength of evidence in this review will be categorized as high, moderate, low, or very low by the GRADEpro software.

\section{Patient and public involvement}

Not applicable. This protocol of systematic review and meta-analysis does not directly involve patients and the general public. Data will be collected from published articles retrieved from main databases and manual search.

\section{Discussion}

Early adequate antimicrobial therapy is crucial for SP management ${ }^{30}$. Besides, anti-inflammatory, anticoagulant, modulating immune states, and other supportive treatments may improve the prognosis of patients with SP. However, inaccurate antibiotic therapy may increase antibiotic resistance, and produce nephrotoxicity and hepatotoxicity. Moreover, the anti-inflammatory properties of glucocorticoids can be used to treat $\mathrm{SP}^{31}$; yet, this type of treatment is associated with some serious side effects. Antibiotics or other medicines used alone are not ideal due to the complexity of SP. Therefore, the optimal strategy has not yet been established.

XBJ is reported to have a positive treatment effect on serious lung infections, including SCAP and sepsis ${ }^{7}$, which suggests that XBJ may also be used to treat SP. Yet, the XBJ-associated side effects remain debatable. E.g., a few studies have suggested that XBJ is a safe treatment approach without serious adverse events 24,32 , while others have reported opposite results ${ }^{33,34}$. The purpose of this metaanalysis is to provide a relatively convincing conclusion of whether XBJ is effective for patients with SP.

The protocol of this review has some potential limitations. Various doses, time points, and duration of XBJ treatment for SP in clinical trials may lead to heterogeneity. For this reason, we plan to perform 
subgroup analyses if data are available. Finally, we will explain the results with caution as well as assess the overall evidence by a critical approach. Conclusions drawn from this review may help update the existing evidence on the benefits and harms of XBJ treatment for SP, thereby benefiting SP patients, clinicians, and policy makers.

\section{Abbreviations}

CAP

community-acquired pneumonia, CENTRAL = Cochrane Central Register of Controlled Trials, CNKI = China National Knowledge Infrastructure, $\mathrm{Cl}=$ confidence interval, $\mathrm{HAP}=$ hospital-acquired pneumonia, $\mathrm{RCT}$ = randomized controlled trials, $\mathrm{SP}=$ severe pneumonia, $\mathrm{VAP}=$ ventilator-associated pneumonia, VIP = China Science and Technology Journal Database, XBJ = xuebijing injection.

\section{Declarations}

Ethics approval and consent to participate: Not applicable.

Consent for publication: Not required

Availability of data and materials: Data sharing is not applicable to this article as no datasets were generated or analyzed during the current study.

Competing interests: None declared

Funding: This study was funded by the National Natural Science Foundation of China (No. 62041701, No. 81973608), and the National Major Scientific and Technological Project (No. 2017ZX10305501).

Author Contributions: QQL proposed the idea of the study. MNZ and LDF designed this study. MNZ drafted the protocol. QQL, LDF, and NL revised the manuscript. MNZ and LDF will search, select, and identify studies included, and then assess the risk of bias of the included studies. SWZ and PJ participated in data extraction and synthesis. Extracted data will be double-checked by PH. QQL will arbitrate any disagreements during the review. All authors have read and approved the publication of the protocol.

Acknowledgements: The authors would like to thank MedSci (www.medsciediting.com) for English language editing.

\section{References}

1. Emergency Physician Branch of Chinese Medical Doctor Association. Expert consensus on clinical practice of emergency severe pneumonia in China. Chinese Journal of Critical Care Medicine. 2016;36. 
2. Müller-Redetzky H, Lienau J, Suttorp N, et al. Therapeutic strategies in pneumonia: going beyond antibiotics. Eur Respir Rev. 2015;24:516 - 24.

3. Simmons J, Pittet JF. The coagulopathy of acute sepsis. Curr Opin Anaesthesiol. 2015;28:227 - 36.

4. Emergency Physician Branch of Chinese Medical Doctor Association. Expert consensus on clinical practice of emergency severe pneumonia in China. Chinese Journal of Critical Care Medicine. 2020;18:122-3.

5. Mizgerd JP. Pathogenesis of severe pneumonia: advances and knowledge gaps. Curr Opin Pulm Med. 2017;23:193-7.

6. De Pascale G, Bello G, Tumbarello M, et al. Severe pneumonia in intensive care: cause, diagnosis, treatment and management: a review of the literature. Curr Opin Pulm Med. 2012;18:213 - 21.

7. Li C, Wang P, Zhang L, et al. Efficacy and safety of Xuebijing injection (a Chinese patent) for sepsis: A meta-analysis of randomized controlled trials. J Ethnopharmacol. 2018;224:512 - 21.

8. Qi F, Liang ZX, She DY, et al. A clinical study on the effects and mechanism of xuebijing injection in severe pneumonia patients. J Tradit Chin Med. 2011;31:46 - 9.

9. Song Y, Yao C, Yao Y, et al. XueBiJing Injection Versus Placebo for Critically III Patients With Severe Community-Acquired Pneumonia: A Randomized Controlled Trial. Crit Care Med. 2019;47:e735$735 \mathrm{e} 743$.

10. Medicine EBOCCC [Xuebijing injection versus placebo for critically ill patients with severe community-acquired pneumonia: a randomized controlled trial: research results and clinical value]. Zhonghua Wei Zhong Bing Ji Jiu Yi Xue. 2019;31:1199 - 203.

11. Chen $X$, Feng $Y$, Shen $X$, et al. Anti-sepsis protection of Xuebijing injection is mediated by differential regulation of pro- and anti-inflammatory Th17 and T regulatory cells in a murine model of polymicrobial sepsis. J Ethnopharmacol. 2018;211:358 - 65.

12. Liu X, Hu Z, Zhou B, et al. Chinese Herbal Preparation Xuebijing Potently Inhibits Inflammasome Activation in Hepatocytes and Ameliorates Mouse Liver Ischemia-Reperfusion Injury. PLoS One. 2015;10:e0131436.

13. Xu T, Zhou L, Shi Y, et al. Metabolomics approach in lung tissue of septic rats and the interventional effects of Xuebijing injection using UHPLC-Q-Orbitrap-HRMS. J Biochem. 2018;164:427 - 35.

14. Gao YL, Chai YF, Yao YM. [Advancement in the research of mechanism of immune dysfunction in sepsis and the regulatory effects of Xuebijing injection]. Zhonghua Shao Shang Za Zhi. 2013;29:1625.

15. Hou SY, Feng XH, Lin CL, et al. Efficacy of Xuebijing for coagulopathy in patients with sepsis. Saudi Med J. 2015;36:164-9.

16. Wang L, Liu Z, Dong Z, et al. Effects of Xuebijing injection on microcirculation in septic shock. J Surg Res. 2016;202:147 - 54 .

17. Yao L, Liu Y, Hou G, et al. Study on xuebijing injection's regulation of immune function in severe pneumonia. Journal of Hubei University of Chinese Medicine. 2017;19:35 - 8. 
18. Gao J, Kong L, Liu S, et al. [A prospective multicenter clinical study of Xuebijing injection in the treatment of sepsis and multiple organ dysfunction syndrome]. Zhonghua Wei Zhong Bing Ji Jiu Yi Xue. 2015;27:465 - 70.

19. Wang L. Analysis of the clinical effect of Xuebijing injection in the treatment of severe pneumonia. Journal of Clinical Pulmonary Medicine. 2018;23:1101-4.

20. Wang D, Zhao L, Li L, et al. Effects of Xuebijing combined with cefoperazone sulbactam sodium and levofloxacin on immune function, coagulation function and therapeutic effect in patients with severe community acquired pneumonia. Chinese Journal of Hospital Pharmacy. 2019;39:1561-5.

21. Zheng $\mathrm{R}$, Wang $\mathrm{H}$, Liu $\mathrm{Z}$, et al. A real-world study on adverse drug reactions to Xuebijing injection: hospital intensive monitoring based on 93 hospitals (31,913 cases). Ann Transl Med. 2019;7:117.

22. Wang C, Shi QP, Ding F, et al. Reevaluation of the post-marketing safety of Xuebijing injection based on real-world and evidence-based evaluations. Biomed Pharmacother. 2019;109:1523-31.

23. Zhu M, Zhang G, Hu M, et al. A systematic review of the curative effect of Xuebijing injection for removing blood stasis and detoxification in the treatment of severe pneumonia. Chinese Journal of Evidence-Based Medicine. 2014;14:462-8.

24. Zhu M, Zhang G, Hu M, et al. A systematic review of the curative effect of Xuebijing injection for removing blood stasis and detoxification in the treatment of severe pneumonia. Chinese Journal of Evidence-Based Medicine. 2020;31:483-9.

25. Feng B, Wang P, Ning Z. A Meta-analysis Xuebijing injection assisted pneumonia cure disease randomized controlled rrials. Tianjin Pharmacy. 2015;27:30 - 3 + 52 .

26. Zhao Y, Yin G, Liu Z, et al. Application of Xuebijing in the treatment of severe pneumonia. China health standards Management. 2019;10:93 - 5.

27. Moher $D$, Shamseer $L$, Clarke $M$, et al. Preferred reporting items for systematic review and metaanalysis protocols (PRISMA-P) 2015 statement. Syst Rev 2015;4:1.

28. Metlay JP, Waterer GW, Long AC, et al. Diagnosis and Treatment of Adults with Community-acquired Pneumonia. An Official Clinical Practice Guideline of the American Thoracic Society and Infectious Diseases Society of America. Am J Respir Crit Care Med. 2019;200:e45-45e67.

29. Guyatt $G H$, Oxman $A D$, Vist GE, et al. GRADE: an emerging consensus on rating quality of evidence and strength of recommendations. BMJ. 2008;336:924-6.

30. Garnacho-Montero J, Barrero-García I, Gómez-Prieto MG, et al. Severe community-acquired pneumonia: current management and future therapeutic alternatives. Expert Rev Anti Infect Ther. 2018;16:667 - 77.

31. Torres A, Sibila O, Ferrer M, et al. Effect of corticosteroids on treatment failure among hospitalized patients with severe community-acquired pneumonia and high inflammatory response: a randomized clinical trial. JAMA 2015;313:677 - 86.

32. Hu J, Shang H, Li J, et al. Xuebijing injection for sepsis: a comprehensive review. Medical Journal of Chinese People's Liberation Army 2010;35:9-12. 
33. Zhao B, Zhang H, Zhao C. Analysis of 198 serious adverse reactions caused by Chinese medicine injection. Guiding Journal of Traditional Chinese Medicine and Pharmacy 2019;25:41 - 3.

34. Guo $\mathrm{H}$, Ren $Y$, Wang P, et al. Literature analysis of adverse reactions of Xuebijing injection. Anhui Medical and Pharmaceutical Journal 2017;21:1548-51.

\section{Figures}

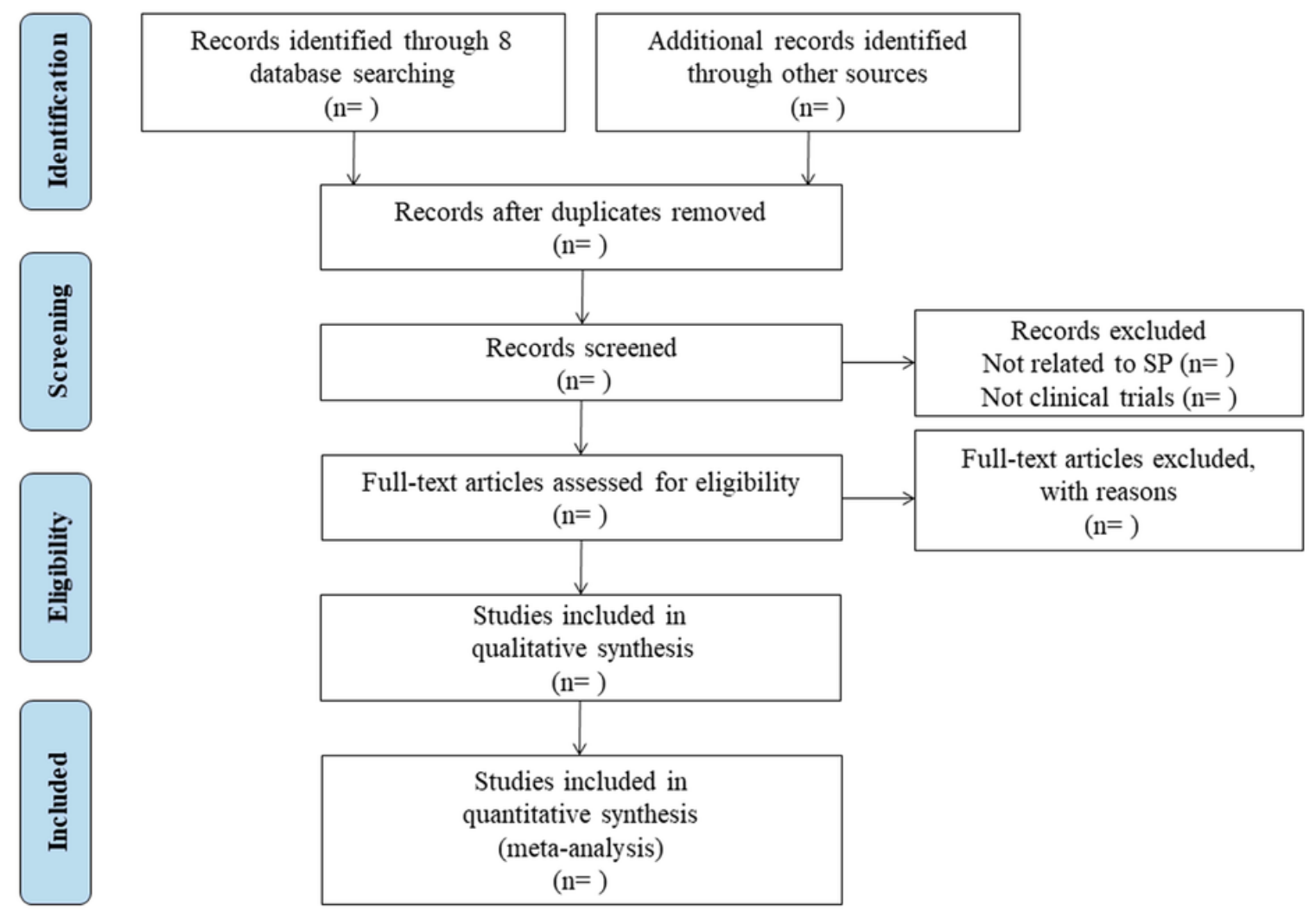

\section{Figure 1}

Flow diagram of the study selection process. SP, severe pneumonia.

\section{Supplementary Files}

This is a list of supplementary files associated with this preprint. Click to download.

- Appendix1.docx

- Appendix2.docx 